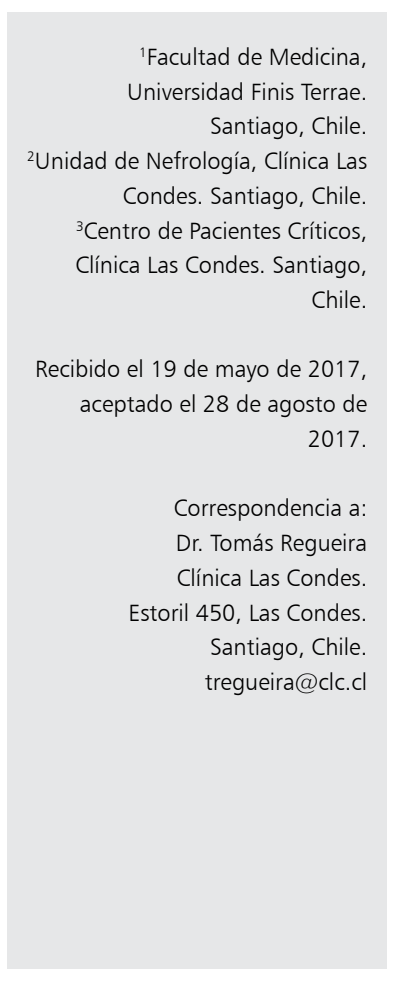

\section{Acidosis láctica asociada a metformina. Caso clínico}

\author{
FELIPE QUINTANA ${ }^{1}$, MARÍA JOSÉ PEZZANI ${ }^{1}$, RODRIGO OROZCO ${ }^{2}$, \\ JORGE DREYSE ${ }^{3}$, LEONARDO SOTO ${ }^{3}$, TOMÁS REGUEIRA ${ }^{3}$
}

\begin{abstract}
Metformin-associated lactic acidosis is a severe and infrequent adverse event. Early diagnosis is essential to start an early treatment, which often has favorable results. We report a 56 years old non-insulin-requiring type 2 diabetic female who developed a severe metabolic acidosis associated with metformin in relation to an acute renal failure secondary to infectious diarrhea. Early treatment with bicarbonate and continuous hemofiltration allowed a quick improvement of the patient. Metformin-associated lactic acidosis has an elevated mortality (50-80\%) and has a specific and effective treatment. Therefore, the condition must be born in mind.
\end{abstract}

(Rev Med Chile 2017; 145: 1072-1075)

Key words: Acidosis, Lactic; Metformin; Renal Insufficiency.

\section{L} a metformina es un agente hipoglicemiante de la familia de las biguanidas que promueve la euglicemia al disminuir la insulino-resistencia, la producción hepática de glucosa y la absorción intestinal de ésta. La acidosis láctica asociada a metformina es una complicación poco frecuente, pero que siempre se debe tener presente a la hora de enfrentar a pacientes que estén cursando con acidosis láctica no asociada a causas evidentes de hipoperfusión tisular. Debe considerarse dada alta prevalencia de DM-2 en nuestra población y requiere de una interrogación dirigida en la anamnesis de cada paciente. Su alta mortalidad, que incluso puede llegar a ser 50$80 \%$, y la existencia de un tratamiento específico y efectivo $^{1}$, son factores que nos obligan a tener una alta sospecha clínica. En este reporte se presenta el caso de una paciente y revisión de la patología.

\section{Caso clínico}

Se trata de una paciente femenina de 56 años, con antecedentes de tabaquismo activo, diabetes mellitus tipo 2 no insulino requiriente (DM2 NIR) e hipertensión arterial (HTA), en tratamiento con losartán $50 \mathrm{mg}$ al día, aspirina $100 \mathrm{mg}$ al día, linagliptina/metformina 2,5/1.000 al día y glibenclamida $5 \mathrm{mg}$ cada $12 \mathrm{~h}$. Acudió al servicio de urgencia por cuadro de 2 días de evolución caracterizado por compromiso del estado general, mialgias generalizadas y disminución del apetito sin descontinuar consumo de fármacos habituales. Un día antes del ingreso se agregaron náuseas, vómitos, que fueron manejadas parcialmente con ondansetrón, y diarrea profusa sin elementos patológicos, asociado a glicemia capilar de $50 \mathrm{mg} / \mathrm{dl}$. Al llegar al servicio de urgencia, se controló nueva glicemia capilar que resultó en $46 \mathrm{mg} / \mathrm{dL}$ (VN $70-100 \mathrm{mg} / \mathrm{dl}$ ), por lo que se inició manejo con 2 ampollas de solución glucosada al $30 \%$ y posteriormente suero glucosado al $10 \%$ en infusión continua. Al examen físico de ingreso destacaba lúcida, sin signos clínicos de hipoperfusión, y con importante dolor abdominal difuso en los 4 cuadrantes, sin clínica de irritación peritoneal. Se realizó ecografía de abdomen que no evidenció lesión intra-abdominal que explicara el cuadro 
clínico. En los exámenes de laboratorio destacaba un ácido láctico elevado de $173 \mathrm{mg} / \mathrm{dL}$ (VN 6,3$18,9 \mathrm{mg} / \mathrm{dl}$ ) asociado a una creatinina de $5,8 \mathrm{mg} /$ $\mathrm{dL}(\mathrm{VN}$ 0,5-0,9 $\mathrm{mg} / \mathrm{dl}$ ) y a un nitrógeno ureico (BUN) de $100 \mathrm{mg} / \mathrm{dL}(\mathrm{VN}<22,4 \mathrm{mg} / \mathrm{dl})$, con hiperkalemia de 6,17 meq/L (VN 3,3-5,1 meq/L).

El dolor abdominal no respondió al tratamiento farmacológico y en forma paralela la paciente presentó deterioro del nivel de conciencia, por lo que se decidió realizar intubación orotraqueal. La tomografía computarizada (TC) de cerebro no evidenció lesiones agudas que pudieran explicar el compromiso de conciencia, solo microangiopatía, y el AngioTC de abdomen y pelvis evidenció colitis difusa inespecífica, sin signos de trombosis venosa o arterial. A su ingreso a la unidad de tratamiento intensivos (UTI), se constata hipertensa, bien perfundida, pero bajo efectos de sedación y bloqueo neuromuscular post intubación. Se solicitó un panel rápido de gases en sangre arterial (GSA) que evidenciaba en un inicio un $\mathrm{pH}$ fuera de rango y luego de 6,8 (VN: 7,35-7,45), el cual se confirmó mediante una nueva muestra en laboratorio de urgencia. Además, se solicitó reacción de polimerasa en cadena (PCR) de patógenos entéricos que resultó positiva para $E$. coli enteropatógena y enteroagregativa, por lo que inició tratamiento antibiótico con ceftriaxona y metronidazol. Destacaba un anion gap de 38 y gap osmolar de 25, por lo que se planteó el diagnostico de acidosis metabólica con anion gap elevado, secundaria a acumulación de ácido láctico probablemente debido a uso de metformina. Se midieron niveles de salicilato, los cuales se encontraban fuera de rango tóxico. La paciente presentó deterioro hemodinámico, manteniendo una adecuada perfusión clínica, con saturación venosa central sobre $65 \%$ y diferencia veno-arterial de $\mathrm{CO}_{2}$ de 5 $\mathrm{mmHg}$, pero con requerimientos de noradrenalina hasta $0,35 \mathrm{mcg} / \mathrm{kg} / \mathrm{h}$ para lograr una presión arterial media de $60 \mathrm{mmHg}$. Se inició el manejo con $500 \mathrm{ml}$ de bicarbonato al 2/3 Molar en $2 \mathrm{~h}$ logrando un $\mathrm{pH}$ 7,1 y luego con hemofiltración de alto volumen (HFAV), logrando normalizar el $\mathrm{pH}(7,4)$ y su situación hemodinámica. $\mathrm{Al}$ día siguiente, su condición clínica mejoró rápidamente, logrando extubarse, suspender drogas vasoactivas y la HFAV (Figura 1).

\section{Discusión}

La acidosis láctica es la causa más común de acidosis metabólica en pacientes hospitalizados ${ }^{2}$. Generalmente se define como concentración de lactato sérico mayor a $4 \mathrm{mmol} / \mathrm{L}(36 \mathrm{mg} / \mathrm{dl})$ y un anion gap $>$ de 12 . Las causas de acidosis láctica se han dividido en 2 grandes grupos: La acidosis láctica tipo $\mathrm{A}$, que se asocia a un mal aporte tisular de oxígeno y la acidosis láctica tipo $\mathrm{B}$, en la cual no existen evidencias de hipoperfusión sistémica. La acidosis metabólica asociada a metformina es un ejemplo de acidosis láctica tipo B.

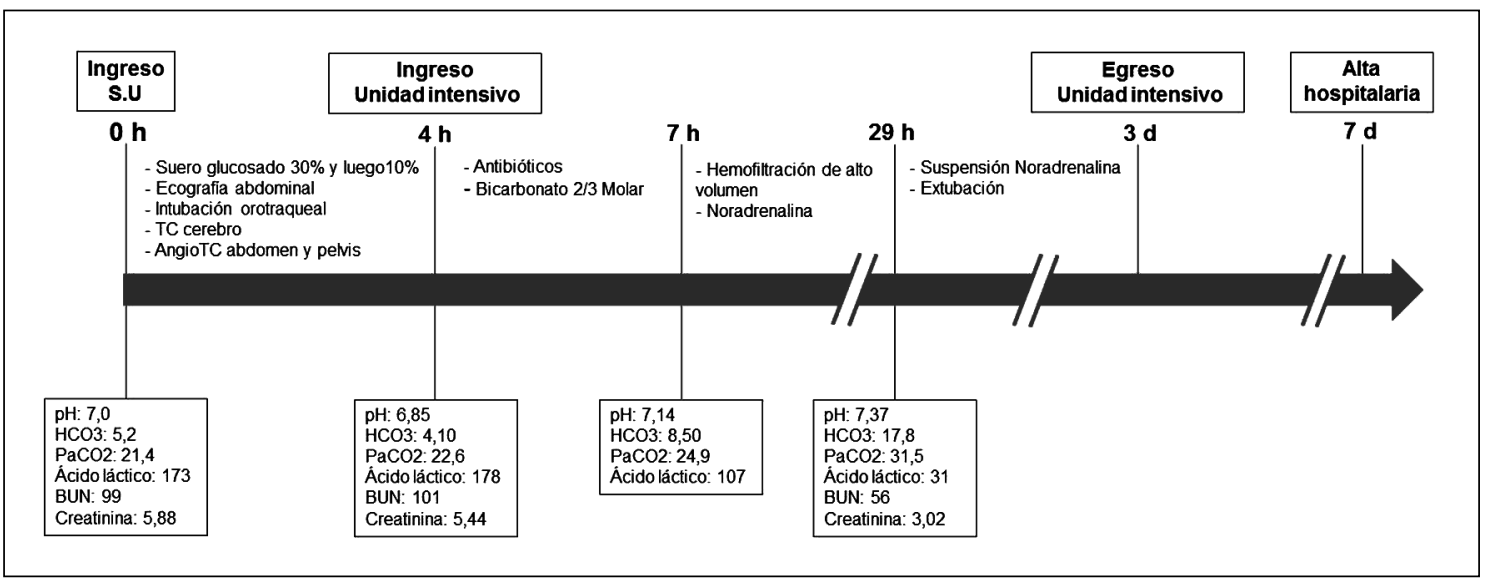

Figura 1. Línea del tiempo en horas $(h)$ y días (d) que evidencia intervenciones realizadas al paciente y evolución de los exámenes de laboratorio. 
La metformina es una biguanida utilizada como fármaco de primera línea en el tratamiento de la diabetes mellitus tipo 2. Se absorbe vía oral, alcanzando un pico de concentración alrededor de las $7 \mathrm{~h}$ ( 4 a $8 \mathrm{~h})$, con una biodisponibilidad del 50 a $60 \%$. Prácticamente no se une a proteínas plasmáticas y su volumen de distribución es alto (358 a $654 \mathrm{~L}$ ). No es metabolizada a nivel plasmático y su excreción es de $90 \%$ vía renal, alcanzando una vida media de eliminación de 6,2 h, que se prolonga en casos de insuficiencia renal. Es un fármaco que se aclara con hemodiálisis. La acidosis láctica secundaria al consumo de este fármaco es un efecto adverso raro, pero importante dado su alta letalidad (50-80\%) 3 . En una revisión de 11.800 pacientes tratados con metformina durante un promedio de 2 años, solo 2 pacientes desarrollaron dicha complicación. Se estima que la incidencia es de 9 casos por cada 100.000 personas expuestas al año ${ }^{4}$.

Se ha observado que las concentraciones plasmáticas elevadas de metformina, como ocurren en individuos con insuficiencia renal, asociado a un evento o condición secundaria que adicionalmente altera la producción de lactato o el aclaramiento de éste (Ejemplo: cirrosis, sepsis o hipoperfusión), son típicamente necesarios para causar esta acidosis láctica asociada con dicho fármaco ${ }^{5}$.

Cabe destacar que se debe tener un alto índice de sospecha clínica de esta causa de acidosis láctica, no solamente por su alta mortalidad, sino también debido al tratamiento que conlleva. Los síntomas son inespecíficos y pueden incluir anorexia, náuseas, vómitos, dolor abdominal, letargia, hiperventilación e hipotensión ${ }^{5}$. En general por tratarse de una acidosis láctica tipo $B$ no se asocia a clínica de hipoperfusión tisular, como en el caso de nuestra paciente. Sin embargo, en estados avanzados no corregidos de acidosis puede ocurrir compromiso de la perfusión ya sea por hipotensión o por compromiso del gasto cardiaco asociado a acidosis sistémica. El uso precoz e interpretación adecuada de los gases arteriales ( $\mathrm{pH}$ y bicarbonato) y electrolitos plasmáticos, permite reconocer el anion gap elevado y descartar con prontitud las causas posibles. La asociación con metformina debe establecerse mediante una adecuada historia clínica, ya que en nuestro medio no existe la posibilidad de medir la concentración plasmática de metformina.

La acidosis láctica asociada a la metformina se produce por el desacoplamiento de la fosforilación oxidativa a nivel de las mitocondrias. La fenformina, otra biguanida que se dejó de usar en DM-2 por la alta incidencia de acidosis láctica, permitió entender mejor la fisiopatología de la acidosis láctica por biguanidas. Estas causan una inhibición de la cadena de fosforilación oxidativa, mayormente a nivel del complejo I. Esto produce acumulación de NADH al interior de la mitocondria que secundariamente inhibe al ciclo de Krebs y promueve la acumulación de piruvato por inhibición de la piruvato deshidrogenasa. La acumulación de piruvato citoplasmático, al exterior de la mitocondria, y de NADH proveniente de la glicolisis anaeróbica, estimulan a la enzima lactato deshidrogenasa a generar lactato. Cuando la producción de lactato sistémico supera la capacidad de utilizar dicho lactato para neoglucogenesis, particularmente en hepatocitos, entonces se genera rápidamente acidosis láctica con repercusiones clínicas significativas. La metformina es menos hidrofóbica que la fenformina y por tanto no logra bloquear en forma habitual la fosforilación oxidativa, excepto al alcanzar concentraciones plasmáticas elevadas como en la insuficiencia renal. La remoción de la metformina y la corrección de la acidosis metabólica se logran en forma adecuada mediante la diálisis (en el caso de esta paciente por hemofiltración continua), que debe ser prolongada dado los riesgos de rebote de la acidosis.

El mejor tratamiento para estos pacientes son el bicarbonato y la hemodiálisis, con los que se puede corregir la acidosis y eliminar la metformina ${ }^{6,7}$. En este caso destaca que la paciente previamente tenía función renal normal y en contexto de una sepsis con deshidratación secundaria a diarrea infecciosa, rápidamente desarrolló esta complicación grave y de alta letalidad. El uso de hemofiltración continua de alto volumen en este caso respondió a la necesidad de utilizar una técnica de reemplazo renal de mayor estabilidad hemodinámica, con una alta tasa de sustitución que permitiese mayor aporte de bicarbonato, para una corrección más rápida del $\mathrm{pH}$ sanguíneo, y en forma continua y de alto volumen para probablemente lograr una mayor remoción de la droga.

Dado la alta prevalencia de diabetes tipo 2 en Chile, estimada en $9,4 \%{ }^{8}$, y siendo la metformina un fármaco de primera línea para esta patología, sugerimos incluir en estos pacientes, en el diagnóstico diferencial de acidosis, la acidosis láctica tipo B asociada al uso de metformina. 


\section{Referencias}

1. Vecchio S, Protti A. Metformin-induced lactic acidosis: no one left behind. Critical Care 2011; 15: 107.

2. Ncomanzi D, Sicat R, Sundararajan K. Metformin-Associated Lactic Acidosis Presenting as an Ischemic Gut in a Patient Who Then Survived a Cardiac Arrest. J Med Case Reports 2014; 8: 159.

3. Gjedde S, Christiansen A, Pedersen S, Rungby J. Survival following a metformin overdose of $63 \mathrm{~g}$ : a case report. Pharmacol Toxicol 2003; 93 (2): 98-9.

4. Defronzo R, Fleming GA, Chen K, Bicsak TA. Metformin-associated lactic acidosis: Current perspectives on causes and risk. Metabolism: Clinical and Experimental 2016; 65 (2): 20-9.
5. Lalau JD. Lactic acidosis induced by metformin: incidence, management and prevention. Drug Saf 2010; 33 (9): 727-40.

6. Lalau JD, Westeel PF, Debussche X, Dkissi H, Tolani M, Coevoet B, et al. Bicarbonate haemodialysis: an adequate treatment for lactic acidosis in diabetics treated by metformin. Intensive Care Med 1987; 13: 383.

7. Heaney D, Majid A, Junor B. Bicarbonate haemodialysis as a treatment of metformin overdose. Nephrol Dial Transplant 1997; 12: 1046.

8. Ministerio de Salud. Encuesta Nacional de Salud 20092010, http://web.minsal.cl/portal/url/item/bcb03d7bc28b64dfe040010165012d23.pdf. [Consultado el 20 de marzo de 2017]. 